\title{
A IMPORTÂNCIA DA SAÚDE VOCAL EM DIFERENTES CATEGORIAS PROFISSIONAIS: uma revisão integrativa
}

\author{
*Luciana Dantas Farias de ANDRADE ${ }^{1}$ \\ Francisco Cleidson Xavier de LACERDA JUNIOR ${ }^{2}$ \\ Heloisy Alves de MEDEIROS ${ }^{3}$ \\ Roberta Lima GONÇALVES ${ }^{4}$
}

\begin{abstract}
${ }^{1}$ Enfermeira. Doutora em Psicologia. Professora da Unidade Acadêmica de Enfermagem, Universidade Federal de Campina Grande, Cuité-PB, Brasil.

${ }^{2}$ Fonoaudiólogo. Especialistaem Saúde da Família pela Universidade Federal da Paraíba, João Pessoa-PB, Brasil.

${ }^{3}$ Enfermeira. Mestre em Saúde Pública. Professora da Unidade Acadêmica de Enfermagem, Universidade Federal de Campina Grande, Cuité-PB, Brasil.

${ }^{4}$ Enfermeira. Mestre em Saúde Pública. Professora da Unidade Acadêmica de Ciências e da Saúde, Universidade Federal de Campina Grande, Campina Grande-PB, Brasil.
\end{abstract}

* Email para correspondência: luciana.dantas.farias@ gmail.com

\section{Recebido em: 22/12/2014 - Aprovado em: 18/06/2015 - Disponibilizado em: 15/07/2015}

\section{Resumo}

O presente artigo aborda uma revisão integrativa da literatura que objetivaenfatizar a importância da saúde vocal em diferentes categorias profissionais. A pesquisa foi do tipo revisão integrativa da literatura realizada no período de setembro a novembro de 2014 na busca às bases de dados indexadorasSciELO e LILACS. Foram utilizados os descritores "fonoaudiologia", "voz" e "prevenção primária", conforme orientação dos Descritores em Ciências da Saúde da Biblioteca Virtual em Saúde com obtenção de artigos envolvendo os anos de 2003 a 2013 copilando oito artigos.De acordo com os artigos analisados pode-se constatar a existência de um número considerável de estudos referente àsaúde vocal de docentes, embora enfatiza-se a importância de aprofundar as pesquisas a outras categorias profissionais que também fazem uso da voz como instrumento de trabalho. Conclui-se que, de fato, os professores são os profissionais que são mais acometidos por distúrbios no aparelho fonador, embora profissionais de saúde, advogados, recreadores, dentre outros, também merecem estudos específicos em suas áreas. A presença do fonoaudiólogo na atenção primária à saúde favorece o desenvolvimento de ações de prevenção/promoção da saúde vocal às diferentes categorias profissionais.
\end{abstract}

Palavras-chave: Fonoaudiologia. Voz. Prevenção Primária.Doenças Profissionais. Pesquisa interdisciplinar.

\begin{abstract}
This article discusses an integrative literature review that aims to emphasize the importance of vocal health in different professional categories. The research was the type integrative literature review carried out from September to November 2014 in the search indexing databases SciELO and LILACS. The key words "speech" were used "voice" and "primary prevention" as orientation of Descriptors in Health Sciences of the Virtual Health Library to obtain articles involving the years 2003 to 2013 compiling eight articles. According to the articles analyzed can- to establish the existence of a considerable number of studies on the vocal health of teachers, while emphasizing the importance of further research to other professional categories that also make use of the voice as a working tool. We conclude that, in fact, teachers are the professionals who are most affected by disturbances in the vocal tract, although health professionals, lawyers, entertainers, among others also deserve specific studies in their areas. The presence of the speech therapist in primary health care favors the development of prevention/promotion of vocal health to the different professional categories.
\end{abstract}

Keywords: Speech, Language and Hearing Sciences.Voice. Primary Prevention. Occupational diseases. Interdisciplinary research. 


\section{Introdução}

A voz é uma identificação individual, que condiz com a estrutura física, sexo e idade. Além desses aspectos, a transmissão da emoção é observável na emissão vocal pelo envolvimento de vias neuromotoras que geram um sentido de inter-relação na comunicação, e se modifica de acordo com a situação e o contexto.(DRAGONE;BEHLAU, 2006)

É inegável que na época atual, vista como a era da informação, a voz assume uma grande importância na maior parte das atividades profissionais, pois acarreta em inúmeros riscos ocupacionais.

Segundo Dragone e Behlau (2006), há mais de uma década as pesquisas brasileiras foram gradualmente aprofundadas na tentativa de compreender a saúde vocal e suas problemáticas.Dessa forma, essas discussõesconstituem-se emsinalizadores do avanço necessário para reorientar as práticas terapêuticas dos fonoaudiólogos.

As reflexões sobre os aspectos relacionados à saúde vocal devem acontecer no sentido de preservar e valorizar a adequada produção da voz, evitando prejuízos no trato vocal. Cada aspecto da saúde vocal precisa sercompreendido isoladamente no contexto da vida de cada indivíduo.

A intervenção com ações preventivas e ampliação do acesso ao conhecimento da saúde vocal reduz consideravelmente esse problema de saúde pública e faz parte do cotidiano laboral dos profissionais de saúde da atenção primária.

Assim sendo, o objetivo do presente artigo é enfatizar a importância da saúde vocal em profissionais de diferentes categorias através da modalidade da revisão integrativa da literatura.

\section{Material e Métodos}

Trata-se de umarevisão integrativaque sumariza pesquisas passadas e tira conclusões globais de um corpo de literatura em particular. Esse tipo de revisão permite a construção de análise ampla, contribuindo para discussões sobre métodos e resultados de pesquisa, assim como reflexões sobre a realização de futuras pesquisas (COMIN; SANTOS, 2010).

Para a operacionalização, foram realizadas as seguintes etapas: seleção da questão temática, estabelecimento dos critérios para a triagem da amostra, análise e interpretação dos resultados e apresentação da revisão. A pesquisa foi realizada no período de setembro a novembro de 2014. Na busca às bases de dados indexadoras SciELO e LILACS foram utilizados os descritores "fonoaudiologia", "voz" e "prevenção primária", conforme orientação do Decs (Descritores em Ciências da Saúde) da Biblioteca Virtual em Saúde (BVS), visualizada no quadro 1 (AQUINO; CHIANCA; BRITO, 2012). 
Quadro 1 - Sistematização da busca eletrônica nos anos de 2000 a 2011

\begin{tabular}{|c|l|c|c|c|}
\hline \multirow{2}{*}{$\begin{array}{c}\text { Banco de } \\
\text { dados }\end{array}$} & \multicolumn{1}{|c|}{ Descritores } & $\begin{array}{c}\text { Artigos } \\
\text { encontrados }\end{array}$ & $\begin{array}{c}\text { Artigos } \\
\text { selecionados }\end{array}$ & $\begin{array}{c}\text { Amostra } \\
\text { final }\end{array}$ \\
\cline { 2 - 5 } & & $\mathbf{N}$ & $\mathbf{n}$ & $\mathbf{n}$ \\
\hline SciELO & Fonoaudiologia & 259 & 0 & 0 \\
\hline SciELO & Prevenção Primária & 206 & 6 & 0 \\
\hline SciELO & Voz & 657 & 0 & 0 \\
\hline SciELO & Fonoaudiologia e Voz & 0 & 0 & 2 \\
\hline SciELO & Prevenção Primária e Voz & 0 & 0 & 0 \\
\hline LILACS & Fonoaudiologia & 942 & 0 & 0 \\
\hline LILACS & Prevenção primária & 61 & 0 & 0 \\
\hline LILACS & Voz & 1550 & 0 & 0 \\
\hline LILACS & Fonoaudiologia e Voz & 0 & 8 & 8 \\
\hline LILACS & Prevenção Primária e Voz & 2125 & & 0 \\
\hline TOTAL & & 0 & & 0 \\
\hline
\end{tabular}

Fonte: Dados da pesquisa.

Foram adotados critérios de inclusão e exclusão para recuperação dos trabalhos. Incluídos aqueles que estivessem publicados na íntegra em idioma vernáculo, formato de artigo científico e que tratassem de relatos de experiência, pesquisas descritivas, pesquisas de campo, revisões integrativas e afins selecionados entre os anos de 2003 e 2013 que tratassem da importância da saúde vocal. Excluídos trabalhos como teses, dissertações, livros e capítulos de livros sendo selecionados 08 artigos científicos, dos quais01 se referia a revisão integrativa e 07 , a pesquisas de campo.

Após a leitura dos resumos e a recuperação dos trabalhos, na íntegra, procedeu-se ao fichamento de acordo com o formulário validado por Ursi (2005) e adaptado às especificidades deste estudo, de modo a dar visibilidade aos principais atributos de cada produção (assunto, idioma, ano de publicação, periódico, autores, local da pesquisa, tipo de publicação, objetivos, número de sujeitos, faixa etária dos sujeitos, critérios de inclusão, tratamento dos dados, intervenções realizadas, resultados e abrangência das conclusões).

Desse modo, os artigos foram apreciados individualmente, segundo suas qualidades científicas. Após essa abordagem preliminar, foi realizada a leitura global do corpus de análise, constituído nas etapas anteriores da revisão integrativa, buscando-se delinear os eixos e 
tendências mais salientes no conjunto do material coligido.

\section{Resultados e discussão}

Inicialmente, foram identificados 657 estudos. Após revisão dos títulos e resumos, consideração dos critérios de inclusão e exclusão, verificação da coerência com a temática pesquisada e eliminação por estarem concomitantemente em mais de uma base de dados, 08 foram efetivamente analisados por referirem nos resultados e/ou conclusões a prática da importância da saúde vocal.

Analisando-se os artigos selecionados (Quadro 1), verificou-se a preocupação de pesquisadores, especialmente a partir do ano de 2008 com assuntos relacionados aos problemas causados no aparelho fonador.

Os artigos asseguram que os hábitos de prevenção, no tocante ao cuidado com a voz, não fazem parte da cultura brasileira. Sendo assim, os profissionais com problemas vocais geralmente só procuram um especialista quando a doença já está bastante avançada.(DRAGONE;BEHLAU, 2006)

Embora a prevenção seja a melhor alternativa para evitar patologias que acometam o aparelho fonador e que esteja diretamente relacionado às atividades laborais dos profissionais da atenção primária, operacionalizada no âmbito da
Estratégia Saúde da Família, não existem estudos que investiguem o acometimento e consequências maléficas deste tipo de afecção nos profissionais de saúde.

Os profissionais estudados nos artigos que são acometidos por alguma disfunção no trato vocal são os professores. No entanto, outros estudos apontam que advogados, telejornalistase recreacionistas também somam as estatísticasreferente a este problema. (DRAGONE, 2011)

Em relação aos professores, sabe-se que a carga horária de trabalho (uso intenso da voz), a falta de hábitos de higiene vocal, a falta de recursos tecnológicos (microfone, caixa amplificada), são fatores que aumentam a probabilidade do desenvolvimento de alterações vocais nesta categoria profissional. (DRAGONE, 2011)

De acordo com as pesquisas realizadas constatou-se que os principais sintomas apresentados por pacientes com problemas relacionados à voz são: rouquidão, ardor na garganta, garganta seca, tosse seca e pigarros. A falta de informação e orientação a profissionais que usam a voz como instrumento de trabalho também influencia o surgimento de problemas no trato vocal. (SILVERIO;et. al.,2008)

Tendo em vista que não existem fonoaudiólogos nas instituições de trabalho e esses sintomas também se assemelham a 
problemas respiratórioscomumente existem dificuldades e equívocos com relação à definição do diagnóstico e tratamento precoce do problema.

Ainda de acordo com as literaturas estudadas, percebeu-se que as principais causas para o desenvolvimento de algum problema no trato vocal tem relação com inúmeras afecções respiratórias, stress e o uso intenso da voz. (XAVIER; SANTOS; SILVA, 2013).

Sabe-se que qualquer problema respiratório(faringite, laringite etc) atingemestruturascomo laringe, faringe, entre outras. Sendo estas importantes para o processo da produção vocal,fazendo assim parte do aparelho fonador. (PENTEADO; RIBAS, 2011).

Em relação ao stress no ambiente de trabalho, alguns fatores como: salas abafadas e quentes, ambiente insalubre com ruídos/barulhos, quantidade de períodos trabalhados e carga horária, quantidade do público alvo trabalhado, podem levar o profissional a um comprometimento emocionale, consequentemente, a um elevado nível de stress, bem como ao uso intenso da voz e mais esforço para falar, comprometendo, assim, o aparelho fonador. 
Quadro 2 - Distribuição dos estudos referente às atividades de educação em saúde segundo identificação do estudo,ano de publicação, tipo de estudo, objetivo, base de dados.

\begin{tabular}{|c|c|c|c|c|}
\hline $\begin{array}{c}\text { Identificação do estudo (autores, } \\
\text { título e periódico) }\end{array}$ & $\begin{array}{c}\text { Ano de } \\
\text { publicação }\end{array}$ & $\begin{array}{l}\text { Tipo de } \\
\text { estudo }\end{array}$ & Objetivo & $\begin{array}{c}\text { Base de } \\
\text { dados }\end{array}$ \\
\hline $\begin{array}{l}\text { Guimarães, Valeriana de Castro; } \\
\text { Viana, Maria Aparecida do Divino } \\
\text { Espiroto Santo Reis; Barbosa, Maria } \\
\text { Alves; Paiva, Maria Luiza de Faria; } \\
\text { Tavares, João Antonio Gomes; } \\
\text { Camargo, Leandro Azevedo de. } \\
\text { Cuidados vocais: questão de } \\
\text { prevenção e saúde. } \\
\text { Revista Ciência\& Saude Coletiva }\end{array}$ & 2010 & $\begin{array}{l}\text { Pesquisa de } \\
\text { campo }\end{array}$ & $\begin{array}{l}\text { Objetivo do estudo } \\
\text { demonstrar resultados } \\
\text { obtidos durante a } 9^{\circ} \\
\text { semana nacional da } \\
\text { voz, realizada no } \\
\text { hospital das clínicas } \\
\text { da Universidade } \\
\text { Federal de Goiás } \\
\text { (UFG) }\end{array}$ & $\begin{array}{l}\text { LILACS } \\
\text { e } \\
\text { SciELO }\end{array}$ \\
\hline $\begin{array}{l}\text { Silverio, Kelly Cristina Alves; } \\
\text { Gonçalves, Claúdia Giglio de } \\
\text { Oliveira; Penteado, Regina Zanella; } \\
\text { Vieira, Tais Pichirilli Guilherme; } \\
\text { Libardi, Aline; Rossi, Daniele. } \\
\text { Ações em saúde vocal: proposta de } \\
\text { melhoria do perfil vocal de } \\
\text { professores. } \\
\text { Revista Pró-Fono de Atualização } \\
\text { Científica. }\end{array}$ & 2008 & $\begin{array}{l}\text { Pesquisa de } \\
\text { campo }\end{array}$ & $\begin{array}{l}\text { Objetivo deste estudo } \\
\text { foi: analisar as } \\
\text { queixas, os sintomas } \\
\text { laríngeos, hábitos } \\
\text { relacionados ao } \\
\text { desmpenho vocal e o } \\
\text { tipo de voz de } \\
\text { professores de uma } \\
\text { escola da rede pública } \\
\text { de ensino antes e após } \\
\text { a participação em } \\
\text { grupos de vivência de } \\
\text { voz }\end{array}$ & LILACS \\
\hline $\begin{array}{l}\text { Penteado, Regina Zanella; Ribas, } \\
\text { Tânia Maestrelli. } \\
\text { Processos educativos em saúde: } \\
\text { análise da literatura da } \\
\text { fonoaudiologia brasileira. } \\
\text { Revista da Sociedade Brasileira de } \\
\text { Fonaudiologia. }\end{array}$ & 2011 & $\begin{array}{l}\text { Revisão } \\
\text { integrativa }\end{array}$ & $\begin{array}{l}\text { Este artigo de revisão } \\
\text { analisa os processos } \\
\text { educativos das ações } \\
\text { coletivas de saúde } \\
\text { vocal do professor } \\
\text { descritas na literatura } \\
\text { fonoaudiológica } \\
\text { brasileira. }\end{array}$ & SciELO \\
\hline $\begin{array}{l}\text { Dragone, Maria Lúcia Oliveira } \\
\text { Suzigan. } \\
\text { Programa de saúde vocal para } \\
\text { educadores: ações e resultados. } \\
\text { Revista CEFAC }\end{array}$ & 2011 & $\begin{array}{l}\text { Pesquisa de } \\
\text { campo }\end{array}$ & $\begin{array}{l}\text { Este artigo descreve } \\
\text { um progrma de saúde } \\
\text { vocal desenvolvido no } \\
\text { perído de } 2002 \text { a } \\
\text { 2005, para educadores } \\
\text { do ensino público } \\
\text { (infantil e } \\
\text { fundamental) do } \\
\text { interior do estado de } \\
\text { São Paulo. }\end{array}$ & SciELO \\
\hline $\begin{array}{l}\text { Ferreira, Léslie Piccolotto; Santos, } \\
\text { Janine Galvão dos; Lima, Maria } \\
\text { Fabiana Bonfim de. } \\
\text { Sintoma vocal e sua provável } \\
\text { causa: levantamento de dados em } \\
\text { uma população. } \\
\text { Revista CEFAC }\end{array}$ & 2009 & $\begin{array}{l}\text { Pesquisa de } \\
\text { campo }\end{array}$ & $\begin{array}{l}\text { Este artigo teve a } \\
\text { finalidade de analisar } \\
\text { a ocorrência de } \\
\text { sintomas vocais e a } \\
\text { relação entre a } \\
\text { presença deles e sua } \\
\text { provável causa na } \\
\text { opinião de uma } \\
\text { amostra populacional } \\
\text { e analisar as } \\
\text { correlações possíveis } \\
\text { entre sintomas e casos } \\
\text { citados. }\end{array}$ & SciELO \\
\hline $\begin{array}{l}\text { Xavier, Ivana Arrais de Lavor } \\
\text { Navarro; Santos, Ana Célia de } \\
\text { Oliveira dos; Silva, Daniele Maria }\end{array}$ & 2013 & $\begin{array}{l}\text { Pesquisa de } \\
\text { campo }\end{array}$ & $\begin{array}{l}\text { Objetivo apresentar } \\
\text { uma ação de } \\
\text { promoção de saúde }\end{array}$ & SciELO \\
\hline
\end{tabular}




\begin{tabular}{|c|c|c|c|c|}
\hline $\begin{array}{l}\text { da. } \\
\text { Saúde vocal do } \\
\text { professor:intervenção } \\
\text { fonoaudiológica na atenção } \\
\text { primária à saúde. } \\
\text { Revista CEFAC }\end{array}$ & & & $\begin{array}{l}\text { vocal dos professores } \\
\text { de três escolas } \\
\text { municipais situadas } \\
\text { no Distrito Sanitário } \\
\text { III, em recife-PE, no } \\
\text { âmbito da atenção } \\
\text { primária à saúde-APS }\end{array}$ & \\
\hline $\begin{array}{l}\text { Santana,Maria da Conceição C. } \\
\text { Pessoa de; Goulart,Bárbara Niegia } \\
\text { Garcia de; Chiari,Brasilia } \\
\text { Maria.Distúrbios da voz em } \\
\text { docentes: revisão crítica da } \\
\text { literatura sobre a prática da } \\
\text { vigilância em saúde do } \\
\text { trabalhador. } \\
\text { Revista Jornal da Sociedade } \\
\text { Brasileira de Fonoaudiologia }\end{array}$ & 2012 & $\begin{array}{l}\text { Revisão } \\
\text { integrativa }\end{array}$ & $\begin{array}{l}\text { Objetivo analisar a } \\
\text { produção } \\
\text { bibliográfica } \\
\text { científica sobre a } \\
\text { prática da vigilãncia à } \\
\text { saúde do trabalhador } \\
\text { relacionados aos } \\
\text { distúrbios vocais em } \\
\text { professores }\end{array}$ & SciELO \\
\hline $\begin{array}{l}\text { Luchesi,Karen Fontes; Mourão,Lucia } \\
\text { Figueiredo; kitamura,Satoshi. } \\
\text { Ações de promoção e prevenção à } \\
\text { saúde vocal de professores: uma } \\
\text { questão de saúde coletiva. } \\
\text { Revista CEFAC }\end{array}$ & 2010 & $\begin{array}{l}\text { Pesquisa de } \\
\text { campo }\end{array}$ & $\begin{array}{l}\text { Objetivo analisar } \\
\text { possíveis ações para } \\
\text { prevenção e a } \\
\text { promoção da saúde } \\
\text { vocal de professores } \\
\text { através de análise de } \\
\text { interações entre } \\
\text { aspectos vocais, } \\
\text { ocupacionais e } \\
\text { preventivos. }\end{array}$ & SciELO \\
\hline
\end{tabular}

Fonte: Dados da pesquisa

Com o objetivo de demonstrar, através desse estudo, a importância e a necessidade da prevenção e promoção da saúde vocal dos profissionais que utilizam a voz como instrumento de trabalho, é de grande importância a realização deintervenções voltadas à sáude desses profissionais com o intuito de amenizar os efeitos do trabalho sobre a sua saúde, bem como a inserção do fonoaudiólogo nas instituições e na atenção primária à saúde a fim de facilitar essas ações na prática cotidiana.

Tendo em vista que estes profissionais não dispõem de prévio treinamento vocal, bem como muitos destes estão inseridos em ambientes de trabalho desfavoráveis à saúde vocal, tornado-osvulneráveisao

desenvolvimento de um problema na voz, faz-se necessário intensificar pesquisas e ações de caráter preventivo, de promoçãoe educação em saúde voltadas ao cuidado com a voz.

\section{Conclusão}

A utilização da revisão integrativa da literatura permitiu a síntese demúltiplos estudos publicados e conduziu as reflexões dos manuscritos a conclusões gerais a respeito do estudo da importância da saúde vocal na modernidade. 
Ficou nítida a importância de ampliar a percepção e análise dos determinantes do processo saúdedoença vocal dos profissionais, deslocando o eixo patologia/tratamento para saúde/promoção, tendo em vista que a maior parte dos estudos realizados enfatiza a patologia adquirida e não a prevenção das mesmas.

Os artigos copilados apontam que a classe mais atingida,dentre osprofissinais, são os professores. Sabese que, de fato, estes profissionais usam a voz como principal recurso de trabalho, além de uma considerável carga horária semanal, um público alvo bastante volumoso, o que requer mais esforço para falar, estão inseridos em um ambiente de trabalho que muitas vezes não disponibiliza recursos tecnológicos que ajudariam a não exigir tanto do aparelho fonador justificando a quantidade de material científico produzido visando a melhora nas estatísticas para esta categoria profissional.

Embora os docentes sejam a categoria profissional mais acometida por patologias no trato vocal, outras categorias também foram enfatizadas nos artigos como os advogados, recreacionistas, telejornalistas, entre outros.

No entanto, a Estratégia Saúde da Família trabalha com atividades preventivas envolvendo atividades de educação em saúde ao indivíduo, família e comunidade, sejam nas consultas individuais, em procedimentos técnicos ou propostas grupais, de modo que os profissionais de saúde também utilizam sobremaneira o aparelho fonador em seu ambiente laboral e nenhum artigo encontrado foi possível elucidar a importância da voz neste contexto.

O que conduz à seguinte reflexão: os profissionais de saúde não são acometidos por problemas no aparelho fonador? Existe a possíbilidade de diagnósticos equivocados levando-os a crer que são comumente acometidos por afecções no aparelho respiratório ou stressno trabalho?

Destarte, sugerem-se estudos de campo que investiguem afecções ao aparelho fonador não só em profissionais de saúde, mas também em advogados, recreacionistas, telejornalistas, entre outras categorias profissionais que também utilizam a voz como instrumento de trabalho. 
Diante do material copilado, as atividades preventivas estariam voltadas para a identificação precoce de riscos associados aos distúrbios vocais, bem como a orientações referente ao comportamento vocal do indivíduo(abuso/uso), além de hábitos, cuidados de higiene/saúde vocal, aquecimento e desaquecimento, exercícios, técnicas vocais, anatomia e fisiologia da produção vocal, motricidade orofacial, entre outras.

Assim, os profissionais desenvolvem a interpretação, significação, vivência da sua voz e relação da mesma com o seu trabalho favorecendo o cuidado com o trato vocal.

De acordo com o estudo realizado percebe-se a importância e a necessidade do profissional de fonoaudiologia em diferentes instituições de trabalho, principalmente no contexto escolar e no ambiente da Atenção Primária à Saúde. Tendo esse profissional que repensar, revisar e reformular as dinâmicas, estratégias e processos educativos por meio dos quais desenvolvem as ações no tocanteà importância da prevenção e promoção da sáude vocal.

\section{Referências}

1 AQUINO, Ana Luiza; CHIANCA, Tânia Couto Machado; BRITO, Renata Cristina Sá Integridade da pele prejudicada, evidenciada por dermatite da área das fraldas: revisão integrativa. Rev. Eletr. Enf. abr./jun., n. 14, v. 2: 414-24, 2012.

2 COMIN, Fábio Scorsolini; SANTOS, Manoel Antônio. O estudo científico da felicidade e a promoção da saúde: revisão integrativa da literatura. Rev. Latino-Am. Enferm. mai/ jun, n. 18, v. 3, 2010 .

3 DRAGONE, Maria Lúcia Suzigan; BEHLAU, Mara. A fonoaudiologia brasileira e a voz do professor: olhares científicas no decorrer do tempo.

Fonoaudiol Bras. n. 4:6-8, 2006.

4 DRAGONE, Maria Lúcia Oliveira Suzigan. Progama de saúde vocal para educadores: ações e resultados. Revista CEFAC. nov/dez, v.13, n.6, 2011.

5 FERREIRA, Léslie Piccolotto; SANTOS, Janine Galvão dos; LIMA, Maria Fabiana Bonfim de. Sintoma vocal e sua provável causa: 
levantamento de dados em uma população. Revista CEFAC. jan/mar, v.11, n.1, 2009.

6 GUIMARÃES, Valeriana de Castro; et.al. Cuidados vocais: questão de prevenção e saúde. Revista Ciência \& Saúde Coletiva.set, v.15, n.6, 2010.

7 LUCHESI, Karen Fontes; MOURÃO, Lúcia Figueiredo; KITAMURA, Satoshi. Ações de promoção e prevenção à saúde vocal de professores: uma questão de saúde coletiva. Revista CEFAC.nov/dez, v.12, n.6, 2010.

8 PENTEADO, Regina Zanella; RIBAS, Tânia Maestrelli. Processos educativos em saúde vocal: análise da literatura da fonoaudiologia. Revista Soc. Bras. Fonoaudiologia. abr/jun, v.16, n.2, 2011.

9 SANTANA, Maria da Conceição C. Pessoa de; GOULART, Bárbara Niegia Garcia de; CHIARI, Brasilia Maria. Distúrbios da voz em docentes: revisão crítica da literatura sobre a prática da vigilância em saúde do trabalhador. Revista Jornal da Sociedade Brasileira de Fonoaudiologia.v.24, n.3, 2012.
10 SILVERIO, Kelly Cristina Alves; et.al. Ações em Saúde Vocal: proposta de melhoria do perfil vocal de professores. Rev Pró-Fono Revista de Atualização Científica.jul/set, v.20, n.3, 2008.

11 URSI, Elizabeth Silva. Prevenção de lesões de pele no perioperatório: revisão integrativa da literatura. 2005. 130 f. Dissertação (Mestrado em Enfermagem) - Ribeirão Preto: Universidade de São Paulo, Escola de Enfermagem de Ribeirão Preto, 2005.

12 XAVIER, Ivana Arrais de Lavor Navarro; SANTOS, Ana Célia Oliveira dos; SILVA, Danielle Maria da Silva. Saúde vocal do professor: intervenção fonoaudiológica na atenção primária à saúde. Revista CEFAC.jul/ago, v.15, n.4, 2013. 